\title{
Editorial
}

\section{Computational Modeling of Physical and Chemical Properties of Nanomaterials}

\author{
Hui Zeng, ${ }^{1,2}$ Meng-Qiu Long, ${ }^{3}$ Ottorino Ori, ${ }^{4,5}$ Mihai V. Putz, ${ }^{5}$ and Jeanlex S. de Sousa ${ }^{6}$ \\ ${ }^{1}$ School of Physics and Optoelectronic Engineering, Yangtze University, Jingzhou, Hubei 434023, China \\ ${ }^{2}$ School of Physics, Nanjing University, Nanjing 210093, China \\ ${ }^{3}$ School of Physics and Electronics, Central South University, Changsha, Hunan 410083, China \\ ${ }^{4}$ Research and Development Division, Actinium Chemical Research, Via Casilina 1626/A, 00133 Rome, Italy \\ ${ }^{5}$ Laboratory of Computational and Structural Physical Chemistry for Nanosciences and QSAR, West University of Timisoara, \\ 300115 Timisoara, Romania \\ ${ }^{6}$ Departamento de Física, Universidade Federal do Ceará, Caixa Postal 6030, 60455-760 Fortaleza, CE, Brazil
}

Correspondence should be addressed to Hui Zeng; zenghui@yangtzeu.edu.cn

Received 30 July 2015; Accepted 30 July 2015

Copyright (c) 2015 Hui Zeng et al. This is an open access article distributed under the Creative Commons Attribution License, which permits unrestricted use, distribution, and reproduction in any medium, provided the original work is properly cited.

Computation modeling of nanomaterials is a powerful theoretical method to explore their fundamental properties. In particular, the practical applications of nanostructured materials rely on the comprehensive understanding of their physical chemistry properties. The computational modeling of nanomaterials has attracted tremendous attention due to its high efficiency on accurate prediction of fundamental properties of nanomaterials, which is crucial to various potential applications such as structural engineering, functionalization of nanomaterials, and computational design. Recently, the development of cluster parallel computer offers powerful computational capabilities to calculate the fundamental properties of complex nanostructured systems and to simulate the physiochemical processes. Recent advances in computational modeling of physical chemistry properties of nanomaterials not only could give us better insight into the underlying mechanisms of physical and chemical reaction processes, but also facilitate the practical applications of nanostructured systems.

As Guest Editors, we have successfully organized this special issue in the past year, which is devoted to conducting current development of computational modeling of physical chemistry properties of nanomaterials. At present, it is our great pleasure to announce the release of this special issue. This special issue covers various aspects relevant to the computational modeling of formation, stability, evolution for the nanostructured system, and accurate prediction of physical chemistry properties. This special issue consists of eight research articles that are contributed from Asian and European scientists.

The work from J. Chuen et al. studied the responses of a quantum wire (QW) connected with wide reservoirs to time-dependent external voltages by using self-consistent manner. Many physical quantities, that is, the distributions of internal potential, the induced charge density, capacitance, and conductance, are computed and their analysis demonstrated that the above physical quantities are mainly dependent on the Fermi energy of systems and the frequency of external voltages. They have shown some resonant peaks in the calculated results of capacitance and conductance versus Fermi energy, and they are originated from the opening of the next higher quantum channels and the oscillations related to the longitudinal resonant electron states of the quantum wires (QW). In addition, the frequency-dependent conductance exhibits two different responses to the external voltages: (a) inductive-like and (b) capacitive-like. Hence, the peaks structure of capacitance is related to the plasmon-like excitation in mesoscopic conductor.

B. Li et al. reported their simulation study on the atomic and electronic structures of single Co atom incorporated with divacancy in armchair graphene nanoribbon (AGNR). The calculated results showed that the Co atom embedded 
in AGNR gives rise to significant impacts on the electronic structures. The presence of the Co doping could introduce magnetic properties. More importantly, their results revealed the arising of spin gapless semiconductor characteristics in both ferromagnetic (FM) and antiferromagnetic (AFM) configurations with doping near the edge, suggesting the robustness for potential application of spintronic. The article by $\mathrm{C}$. Cao et al. has investigated the spin-dependent electronic transport properties of zigzag-edged germanium nanoribbons (ZGeNRs). Their calculations revealed that the even 6-ZGeNR has a dual spin-filter effect in antiparallel (AP) magnetism configuration, while the odd 5-ZGeNR behaves as conventional conductor with linear currentvoltage dependence. The magnetoresistance of the $6-\mathrm{ZGeNR}$ can be efficiently modulated by means of tuning the spin configurations (namely, the parallel and antiparallel) of the two electrodes, and the magnetoresistance efficiency is expected to achieve $270 \%$ by switching the magnetic configurations of the electrodes.

Y. Xiao and colleagues have theoretically and numerically studied the effects of dielectric environment on phase resonance in compound grating. They have demonstrated that the transmission characteristics can be tailored by changing the dielectric environment. In addition, the electric field for the peak at the shorter wavelength of the dip is mainly concentrated on the slit filled with smaller dielectric constant, while the electric field is located on the slit filled with larger dielectric constant for the peak at the longer wavelength of the dip. By selecting a proper dielectric environment, the channel selecting device can be constructed by using the dielectric compound grating.

A. Greco and A. Maffezzoli reported an finite element (FE) analysis on the diffusion in 3D nanocomposites obtained with stacks of lamellar nanofillers characterized by the presence of permeable galleries. Their developed model is able to account for diffusion between stacks (interstack diffusion) as well as diffusion inside stacks (intrastack diffusion). Simulation results showed that intrastack diffusion, related to flow inside galleries, can be quite relevant, particularly at high values of gallery thickness. They have shown that the diffusion behavior in intercalated nanocomposites is not well predicted without the consideration of intrastack diffusion effect. The intrastack diffusivity is shown to depend on the morphological features of the nanofiller requiring the development of a proper mathematical model. M. Yang et al. proposed a comprehensive model that incorporates the essential mechanisms of pyrolysis based on the phase field framework, which aimed at better understanding of the morphological evolution in pyrolysis. The pyrolysis is recently proposed as an efficient fabrication technique of micro/nanoscale carbon structures. Their computational analysis with the developed model provides information about the effect of interface energy and kinetic rate on the morphological evolution in pyrolysis. The calculated results could be helpful to design the final shape of carbon structure.

Q. Xiong and X. Tian's work studied the mechanical properties of carbon nanotube (CNT) reinforced polyethylenes (PE) with interfacial covalent bonded interaction by using molecular dynamics simulations. The Young modulus and the interfacial shear stress of the nanoreinforced polyethylene are obtained through a series of the tensile and pullout tests of carbon nanotube reinforced polyethylene. The comparisons between the MD results of their work with the relevant experimental data have shown that the interfacial covalent bonded interaction between CNTs and the polymer matrix is indispensable. The bond interaction plays the main role in the load transfer of nanocomposites. Accurate stressstrain curves have been derived for single- and multiwalled carbon nanotubes enabling future applicative developments of CNTs as nanoreinforcements. In addition, the influences of carbon nanotube embedded length and diameter on the interfacial mechanical properties are also evaluated. V. K. Pustovalov and L. G. Astafyeva conducted an investigation on the core-shell nanoparticles with oxide shell from core metal. The formation of oxide shell on metallic nanoparticles can be achieved by different chemical and physical methods including also natural oxidation of pure metallic nanoparticles in gaseous or liquid media, containing oxygen components (air, water, etc.). We numerically calculated efficiency factors of absorption $K_{\text {abs }}$, scattering $K_{\text {sca }}$, and extinction $K_{\text {ext }}$ of radiation with wavelength $\lambda$ in the spectral interval 150$1000 \mathrm{~nm}$ by spherical homogeneous metallic and two-layered (metal core oxide metal shell) nanoparticles: $\mathrm{Al}, \mathrm{Al}-\mathrm{Al}_{2} \mathrm{O}_{3}$; $\mathrm{Zn}, \mathrm{Zn}-\mathrm{ZnO}$ with core radii in the range $5-50 \mathrm{~nm}$ and shell thickness $5 \mathrm{~nm}$.

We sincerely hope that this special issue can provide a valuable reference to the recent advances and future perspective of computational modeling of physical and chemical properties of nanomaterials. In the end, we also hope that the collection of these articles will inspire both scientific and technological researchers and stimulate innovations for material sciences and related fields.

\section{Acknowledgments}

As Guest Editors, we are grateful to all of the above authors for their contributions to this special issue. In the meantime, we also would like to give thanks to every Reviewer for his/her solid work that improved the quality of these manuscripts.

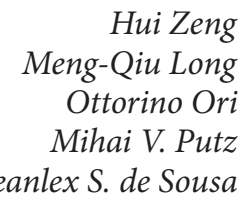



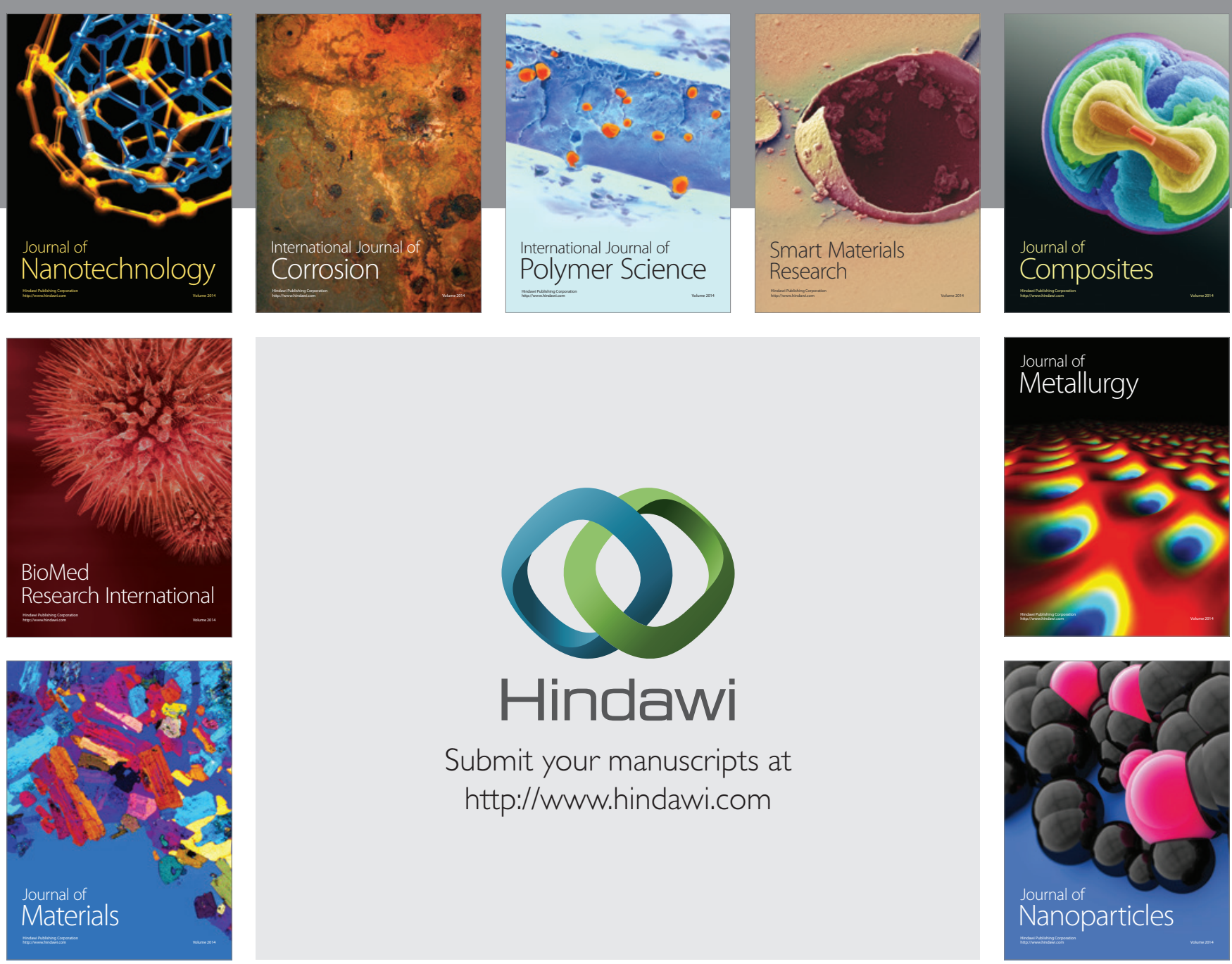

Submit your manuscripts at http://www.hindawi.com
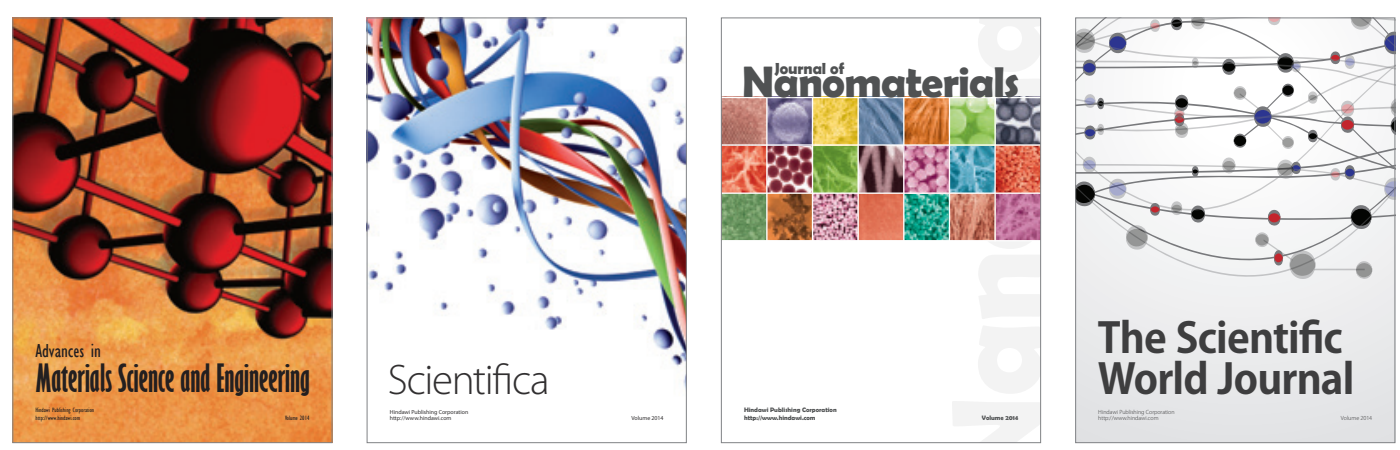

\section{The Scientific World Journal}
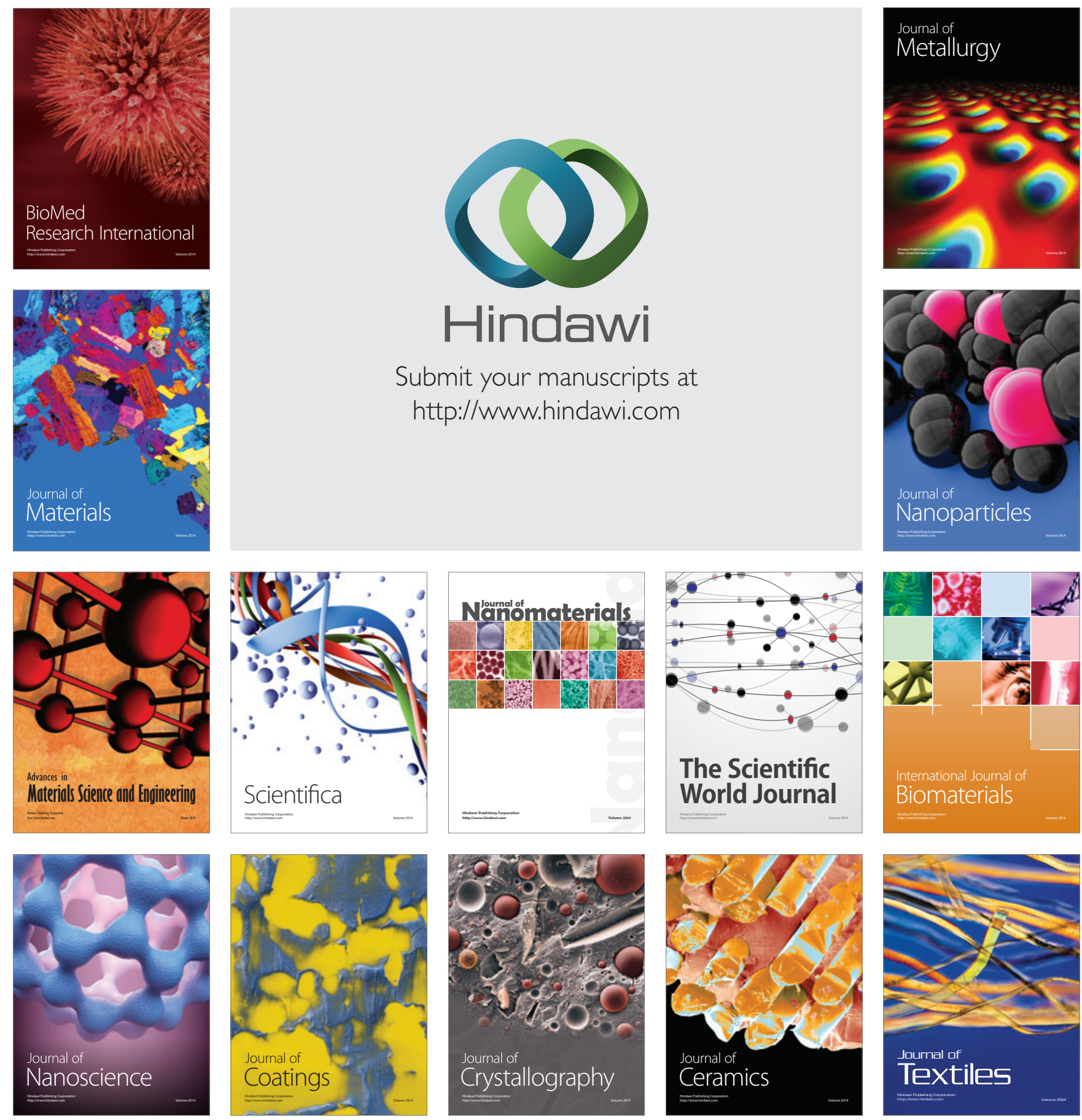